\title{
新型 2-芳基-4-烷基噻唑甲酸衍生物的设计、合成与生物活性研究
}

\author{
朱有全* 王丹阳 袁燕伟 马 源邹小毛* 杨华铮
}

(南开大学元素有机化学研究所 元素有机化学国家重点实验室 天津 300071)

\begin{abstract}
摘要 为了发现具有良好生物活性的新型先导化合物, 通过活性亚结构拼接方法, 在保留噻唑菌胺、甲噻灵和噻氟菌 胺等中的 1,3-噻唑-5-羰基活性部分, 将具有良好生物多样性的苯氧乙酸酯基团引入, 设计合成了一系列含苯氧乙酸酯

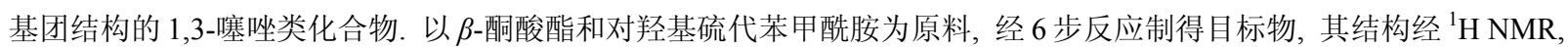
IR 及高分辨质谱确证，并对该类化合物合成方法进行了探讨. 初步生测结果表明，部分化合物对小麦赤霉表现出明显 的抑制活性，在 $50 \mu \mathrm{g} / \mathrm{mL}$ 浓度下对小麦赤霉的抑制率达到 $52 \%$.
\end{abstract}

关键词 1,3-噻唑; 活性亚结构; 苯氧乙酸酯; 噻氟菌胺; 杀菌活性; 除草活性

\section{Design, Synthesis and Bioactivity of Novel 2-Aryl-4-alkylthia- zole-5-carboxylic Acid Derivatives}

\author{
Zhu, Youquan* Wang, Danyang Yuan, Yanwei Ma, Yuan \\ Zou, Xiaomao* Yang, Huazheng \\ (State Key Laboratory of Elemento-organic Chemistry, Institute of Elemento-organic Chemistry, \\ Nankai University, Tianjin 300071)
}

\begin{abstract}
To find new lead compounds with muti-bioactivities, a series of novel 1,3-thiazole-5-carboxylic acid derivatives containing aryloxyacetate were designed via the method of linking active sub-structures, in which the aryloxyacetate with multi-bioactivity was combined to the 1,3-thiazole moiety of ethaboxam, metsulfovax and thifluzamide. The target compounds were synthesized from $\beta$-keto acid ester and 4-hydroxybenzothioamide in 6 steps. Their structures were confirmed by ${ }^{1} \mathrm{H}$ NMR, IR and HRMS techniques. The preliminary bioassay results indicated that some compounds showed obvious inhibition effects against Fusarium graminearum, and the fungicidal activity of $\mathbf{1 j}$ is $52 \%$ at $50 \mu \mathrm{g} / \mathrm{mL}$.
\end{abstract}

Keywords 1,3-thiazole; active sub-structure; phenoxyacetate; thifluzamide; fungicidal activity; herbicidal activity

1,3 -噻唑类衍生物作为含氮杂环化合物的一员在农 药和医药中有着特殊的地位, 其衍生物的优异生物活性 更是吸引了许多农药化学家的注意, 不断有新型的 1,3噻唑衍生物被研究和开发, 部分化合物已被开发成商品 化农用化学品品种或新型先导化合物, 如新烟碱类杀虫 剂噻虫溙 ${ }^{[1]}$ 和噻虫胺 ${ }^{[1]}$ 、用于防治卵菌纲病原菌引起的 葡萄霜霉病和马铃薯晚疫病等病害的噻唑杀菌剂噻唑 菌胺 ${ }^{[2]}$ 、噻氟菌胺 ${ }^{[3]}$ 和新型噻唑类杀菌化合物 $\mathbf{A}^{[4]}$ 、在 $20 \mathrm{~g} / \mathrm{ha}$ 可完全防治稗草的化合物 $\mathbf{B}\left(\right.$ 图 1) ${ }^{[5]}$. 这些研究结 果表明，1,3-噻唑环通过采用不同的连接方式与多种取 代基进行连接形成的 1,3-噻唑类衍生物构成了具有生物
活性多样性农药品种的一个重要分支, 同时也表明 1,3噻唑环是一非常重要的活性结构片段, 继续对 1,3-噻唑 类衍生物合成方法与生物活性研究具有非常重要的意 义. 在结构上同时我们也注意到取代苯氧乙酸类化合物 C 是一类较早开发且目前还在广泛使用的一类植物生 长调节和除草剂品种(如 2,4-二氯苯氧乙酸酯类衍生物) (图 1) ${ }^{[6,7]}$, 为了获得具有生物多样性的新型品种或先导 化合物, 我们根据活性亚结构拼接理论, 基于噻唑菌胺、 噻氟菌胺、新型噻唑类杀菌化合物 $\mathbf{A}$ 、除草品种 $\mathbf{B}$ 和 $\mathbf{C}$ 的结构特点, 在 1,3 -噻唑环结构中引入苯氧乙酸活性 结构, 设计并采用下列路线合成了化合物 1 (Scheme 1),

\footnotetext{
*E-mail: zyq8165@nankai.edu.cn

Received July 4, 2012; revised July 16, 2012; published online July 19, 2012.

Project support by the National Natural Science Foundation of China (Nos. 20772066, 21072108), the National Basic Research Program of China (973 Program) (No. 2010CB126103) and the National Key Technologies R\&D Program (No. 2011BAE06B05-3).

国家自然科学基金(Nos. 20772066, 21072108)、国家 973 计划(No. 2010CB126103)和 “十二五” 科技支撑计划(No. 2011BAE06B05-3)资助项目。
} 
<smiles>CNC(NCc1cnc(Cl)s1)[N+](=O)[O-]</smiles>

噻虫胺<smiles>C[14CH3]</smiles>

噻虫嗪<smiles>CCNc1nc(CC)c(C(=O)NC(C)(CC)c2cccs2)s1</smiles>

噻唑菌胺

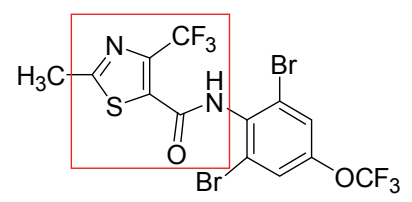

噻氟菌胺<smiles>[Y1]C(=O)COc1ccc([R])cc1[R]</smiles>

图 1 一些商品化品种的结构式

Figure 1 Structures of some commercial products

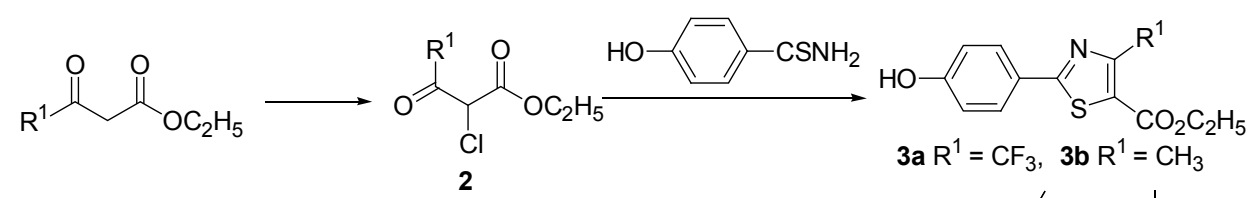<smiles>CCOC(=O)COc1ccc(-c2nc(C(F)(F)F)c(C(=O)OCC)s2)cc1</smiles><smiles>[R]OC(=O)COc1ccc(-c2nc([R])c(C(=O)O[R])s2)cc1</smiles>

1b $R^{1}=\mathrm{CH}_{3}, \mathrm{R}^{2} \mathrm{X}=\mathrm{OCH}_{3}, \mathrm{R}^{3}=\mathrm{CH}_{3} ; \mathbf{1 c} \mathrm{R}^{1}=\mathrm{CH}_{3}, \mathrm{R}^{2} \mathrm{X}=\mathrm{OCH}_{3}, \mathrm{R}^{3}=\mathrm{C}_{2} \mathrm{H}_{5} ; \mathbf{1 d} \mathrm{R}^{1}=\mathrm{CH}_{3}$, $R^{2} X=O_{3}, R^{3}=\mathrm{C}_{4} \mathrm{H}_{9} ; 1$ e R $\mathrm{R}^{1}=\mathrm{CH}_{3}, \mathrm{R}^{2} \mathrm{X}=\mathrm{OCH}_{3}, \mathrm{R}^{3}=\mathrm{C}_{8} \mathrm{H}_{17} ;$ if $\mathrm{R}^{1}=\mathrm{CH}_{3}, \mathrm{R}^{2} \mathrm{X}=\mathrm{OC}_{2} \mathrm{H}_{5}$, $\mathrm{R}^{3}=\mathrm{CH}_{3} ; \mathbf{1 g ~ R} \mathrm{R}^{1}=\mathrm{CH}_{3}, \mathrm{R}^{2} \mathrm{X}=\mathrm{OC}_{2} \mathrm{H}_{5}, \mathrm{R}^{3}=\mathrm{C}_{2} \mathrm{H}_{5} ; \mathbf{1 h} \mathrm{R}^{1}=\mathrm{CH}_{3}, \mathrm{R}^{2} \mathrm{X}=\mathrm{OC}_{2} \mathrm{H}_{5}, \mathrm{R}^{3}=\mathrm{C}_{4} \mathrm{H}_{9} ; \mathbf{1 i}$ $\mathrm{R}^{1}=\mathrm{CH}_{3}, \mathrm{R}^{2} \mathrm{X}=\mathrm{OC}_{2} \mathrm{H}_{5}, \mathrm{R}^{3}=\mathrm{C}_{8} \mathrm{H}_{17} ; \mathbf{1} \mathrm{R}^{1}=\mathrm{CH}_{3}, \mathrm{R}^{2} \mathrm{X}=i-\mathrm{C}_{3} \mathrm{H}_{7} \mathrm{NH}, \mathrm{R}^{3}=\mathrm{CH}_{3} ; \mathbf{1} \mathbf{k} \mathrm{R}^{1}=\mathrm{CH}_{3}$, $\mathrm{R}^{2} \mathrm{X}=i-\mathrm{C}_{3} \mathrm{H}_{7} \mathrm{NH}, \mathrm{R}^{3}=\mathrm{C}_{4} \mathrm{H}_{9} ; 1 / \mathrm{R}^{1}=\mathrm{CH}_{3}, \mathrm{R}^{2} \mathrm{X}=s-\mathrm{C}_{4} \mathrm{H}_{9} \mathrm{NH}, \mathrm{R}^{3}=\mathrm{C}_{8} \mathrm{H}_{17} ; 1 \mathrm{~m} \mathrm{R}^{1}=\mathrm{CF}_{3}, \mathrm{R}^{2} \mathrm{X}=$ $i-\mathrm{C}_{3} \mathrm{H}_{7} \mathrm{NH}, \mathrm{R}^{3}=\mathrm{CH}_{3} ;$ in $\mathrm{R}^{1}=\mathrm{CF}_{3}, \mathrm{R}^{2} \mathrm{X}=i-\mathrm{C}_{3} \mathrm{H}_{7} \mathrm{NH}, \mathrm{R}^{3}=\mathrm{C}_{2} \mathrm{H}_{5} ; 1$ o $\mathrm{R}^{1}=\mathrm{CF}_{3}, \mathrm{R}^{2} \mathrm{X}=$ $i-\mathrm{C}_{3} \mathrm{H}_{7} \mathrm{NH}, \mathrm{R}^{3}=\mathrm{C}_{8} \mathrm{H}_{17} ; 1 \mathrm{p} \mathrm{R}^{1}=\mathrm{CF}_{3}, \mathrm{R}^{2} \mathrm{X}=\mathrm{s}-\mathrm{C}_{4} \mathrm{H}_{9} \mathrm{NH}, \mathrm{R}^{3}=\mathrm{CH}_{3} ; \mathbf{1 q} \mathrm{R}^{1}=\mathrm{CF}_{3}, \mathrm{R}^{2} \mathrm{X}=\mathrm{OCH}_{3}$, $\mathrm{R}^{3}=\mathrm{CH}_{3} ; 1 \mathrm{r} \mathrm{R}^{1}=\mathrm{CF}_{3}, \mathrm{R}^{2} \mathrm{X}=\mathrm{OCH}_{3}, \mathrm{R}^{3}=\mathrm{C}_{4} \mathrm{H}_{9}$

\section{Scheme 1}

探讨该类杂环化合物的合成方法和生物活性.

\section{1 结果与讨论}

以 $\beta$-酩酸酯和对羟基硫代苯甲酰胺为原料合成目 标化合物 1 , 可通过如下 2 种途径实现.

方法一: 以中间体 3 或 7 为原料, 在无水碳酸钾存 在下，与溴乙酸乙酯反应得化合物 $\mathbf{4}$, 然后在碱作用下 将乙酸酯基水解成酸 $\mathbf{5}$, 再分别与二氯亚砜、醇或胺反 应制得化合物 1 (Scheme 1). 由于化合物 4 分子结构中
同时含有两个酯基，碱水解时就要考虑到酯水解的选向 问题, 要保证噻唑环上的酯基不水解, 这就需要探索水 解时所需要的碱液的浓度和温度条件, 经过多次研究发 现采用 $1 \sim 1.5 \mathrm{~mol} \cdot \mathrm{L}^{-1}$ 浓度的氢氧化钠溶液, 且温度保 持在 $20 \sim 25{ }^{\circ} \mathrm{C}$ 时才能保证噻唑环上的酯基不水解, 否 则碱液浓度增加或温度升高, 均会导致噻唑环上的酯基 的水解，这是实验不想看到的情况. 而当噻唑环上的酯 基变换为酰胺基时, 反应温度范围 $\left(<40{ }^{\circ} \mathrm{C}\right)$ 和氢氧化 钠的浓度 $\left(<3 \mathrm{~mol} \cdot \mathrm{L}^{-1}\right)$ 均可提高, 进而加速反应的进行. 
方法二：将中间体 $\mathbf{3}$ 在碱作用下，水解为化合物 $\mathbf{6}$, 再分别与二氯亚砜、醇或胺反应制得中间体 7 , 然后与 由乙酰氯制备的各种中间体进行缩合制备目标产物 $\mathbf{1}$ (Scheme 1).

虽然以上 2 种方法在总的合成步骤方面均为 6 步且 方法非常相近, 但是与方法一相比, 方法二具有易于操 作、收率高的特点, 每一步副反应少、产物明确的优点. 而方法一中在合成噻唑环上连有酯基的中间体 5 时, 容 易得到两个酯基均被水解的产物. 由于方法二最后的缩 合步骤需要在碱性条件下 $80{ }^{\circ} \mathrm{C}$ 以上反应, 因而不适于 合成对高温或碱敏感的 $R^{2} X$ 或 $R^{3}$ 衍生物, 方法一的成 功为我们今后合成化合物 1 类似物提供了多种途径. 由 于我们设计得化合物 1 分子中不含有对高温或碱敏感的 $\mathrm{R}^{2} \mathrm{X}$ 或 $\mathrm{R}^{3}$ 的基团，除化合物 4 和 $1 \mathrm{a}$ 外，均采用方法二进 行合成.

图谱解析：以化合物 $\mathbf{1 d}$ 为例, $\delta 0.94$ 处是丁基上的 甲基氢, $\delta 1.33 \sim 1.41$ 和 $1.62 \sim 1.70$ 处是丁基链上两个亚 甲基氢, $\delta 2.79$ 处是噻唑环上的甲基氢, $\delta 3.91$ 处是噻唑 环上甲氧基氢, $\delta 4.24$ 处是丁基上与氧相连的亚甲基氢, $\delta 4.71$ 处是苯环上羰基和氧之间的亚甲基氢, $\delta 6.98$ 处和 7.93 处是苯环上的四个氢. 该化合物分子中的两个羰基 红外伸缩振动吸收峰位于 1760 和 $1715,834 \mathrm{~cm}^{-1}$ 表明苯 环是对位取代.

构效关系讨论: 目标化合物的除草活性见表 1 及部 分化合物杀菌活性见表2. 由表中的生测数据可知, 当 $\mathrm{R}^{2} \mathrm{X}={ }_{s}-\mathrm{C}_{4} \mathrm{H}_{9} \mathrm{NH}, i-\mathrm{C}_{3} \mathrm{H}_{7} \mathrm{NH} ; \mathrm{R}^{1}=\mathrm{C}_{4} \mathrm{H}_{9}, \mathrm{C}_{8} \mathrm{H}_{17}$ 时, 该系类 化合物对稗草有一定的除草活性而对油菜没有除草活 性, 例如: $1 \mathbf{j}$ 和1q. 相反, 当 $\mathrm{R}^{2} \mathrm{X}=\mathrm{OCH}_{3}, \mathrm{OC}_{2} \mathrm{H}_{5} ; \mathrm{R}^{3}=$ $\mathrm{CH}_{3}, \mathrm{C}_{2} \mathrm{H}_{5}, \mathrm{C}_{4} \mathrm{H}_{9}$ 时该系类化合物对油菜有活性而对稗草 几乎没有活性, 例如: 1a, 1b, 1e, 1g, 1o, 1m. 同时发现 $\mathrm{R}^{1}=\mathrm{CH}_{3}, \mathrm{R}^{2} \mathrm{X}=\mathrm{OC}_{2} \mathrm{H}_{5}, \mathrm{R}^{3}=\mathrm{CH}_{3}$ 所得到的化合物 $\mathbf{1 f}$ 与 $\mathrm{R}^{1}=\mathrm{CF}_{3}, \mathrm{R}^{2}=\mathrm{CH}_{3} \mathrm{O}, \mathrm{R}^{3}=\mathrm{C}_{4} \mathrm{H}_{9}$ 所得到的化合物 $1 \mathbf{r}$, 其对 稗草和油菜的除草活性显著高于其他化合物. 对部分化

表 1 化合物 $1 \mathrm{a} \sim 1 \mathrm{r}$ 的除草活性(抑制率/\%)

Table 1 Herbicidal activities of compounds $1 \mathbf{a} \sim \mathbf{1 r}$ (inhibiting rate/\%)

\begin{tabular}{ccc||ccc}
\hline Compd. & 油菜 & 稗草 & Compd. & 油菜 & 稗草 \\
\hline $\mathbf{1 a}$ & 10 & 0 & $\mathbf{1 j}$ & 0 & 40 \\
$\mathbf{1 b}$ & 18 & 0 & $\mathbf{1 k}$ & 0 & 0 \\
$\mathbf{1 c}$ & 0 & 25 & $\mathbf{1 l}$ & 3 & 12 \\
$\mathbf{1 d}$ & 0 & 0 & $\mathbf{1 m}$ & 10 & 0 \\
$\mathbf{1 e}$ & 5 & 0 & $\mathbf{1 n}$ & 0 & 5 \\
$\mathbf{1 f}$ & 40 & 20 & $\mathbf{1 0}$ & 14 & 0 \\
$\mathbf{1 g}$ & 30 & 0 & $\mathbf{1 p}$ & 0 & 0 \\
$\mathbf{1 h}$ & 0 & 0 & $\mathbf{1 q}$ & 0 & 25 \\
$\mathbf{1 i}$ & 0 & 0 & $\mathbf{1 r}$ & 22 & 20 \\
\hline
\end{tabular}

表 2 部分化合物的杀菌活性(抑制率/\%)

Table 2 The fungcidal activities of compounds $\mathbf{1 a} \sim \mathbf{1 r}$ (inhibiting rate/\%)

\begin{tabular}{cccccc}
\hline Compd. & $\begin{array}{c}\text { 黄瓜 } \\
\text { 枯萎 }\end{array}$ & $\begin{array}{c}\text { 苹果 } \\
\text { 轮纹 }\end{array}$ & $\begin{array}{c}\text { 番茄 } \\
\text { 早疫 }\end{array}$ & $\begin{array}{c}\text { 花生 } \\
\text { 褐斑 }\end{array}$ & $\begin{array}{c}\text { 小麦 } \\
\text { 赤霉 }\end{array}$ \\
\hline $\mathbf{1 c}$ & 5 & 14 & 6 & 19 & 0 \\
$\mathbf{1 f}$ & 10 & 14 & 0 & 7 & 0 \\
$\mathbf{1 g}$ & 0 & 15 & 0 & 13 & 20 \\
$\mathbf{1 j}$ & 0 & 14 & 0 & 7 & 52 \\
$\mathbf{1 k}$ & 10 & 0.0 & 7 & 9 & 0 \\
$\mathbf{1 1}$ & 5 & 10 & 5 & 8 & 16 \\
$\mathbf{1 m}$ & 0 & 16 & 16 & 19 & 0 \\
$\mathbf{1 r}$ & 0 & 24 & 11 & 25 & 0 \\
\hline
\end{tabular}

合物 1 做了杀菌活性测试, 生测结果表明化合物 $\mathbf{1 j}$ 对小 麦赤霉的抑制率达到 52\%, 虽然没发现特别高活性的化 合物，但是该部分工作为进一步研究噻唑类衍生物的生 物活性奠定了一定工作基础.

\section{2 结论}

本论文为了发现具有良好生物活性的新型先导化 合物, 通过活性亚结构拼接方法, 在 1,3-噻唑环 2-位引 入具有良好生物多样性的 4-烷氧羰基甲氧基苯基官能 结构, 设计合成了一系列含苯氧乙酸酯基团结构的 1,3噻唑类化合物，并对该类化合物合成方法进行了探讨. 初步生测结果表明, 部分化合物对小麦赤霉表现出明显 的抑制活性，在 $50 \mu \mathrm{g} \cdot \mathrm{mL}^{-1}$ 浓度下对小麦赤霉的抑制率 达到 $52 \%$.

\section{3 实验部分}

\section{1 仪器与试剂}

北京科仪电光仪器厂XT 4A 显微熔点测定仪, 温度 计未校正; Varain Mercury Plus $400 \mathrm{MHz}$ 核磁共振仪, TMS 为内标; Shimadzu-IR 435 红外光谱仪, 澳化钾压 片; 德国产 Elementar vario EL 元素分析仪; 高分辨质 谱: Varian 7.0T FTICR-MS-ESI. 实验用试剂和原料均为 分析纯或化学纯, 并根据需要进行了常规处理. 参照文 献[8]报道的方法合成化合物 2-氯-4,4,4-三氟-3-氧代丁 酸乙酯(2a). 参照文献[9]报道的方法合成化合物 4-(5-乙 氧羰基-4-甲基噻唑-2-基)苯酚(3b).

\section{2 实验方法}

1.2.14-(5-乙氧羰基-4-三氟甲基噻唑-2-基)苯酚(3a) 的制备

参照合成化合物 $\mathbf{3 b}$ 的类似方法合成化合物 $\mathbf{3} \mathbf{a}^{[9]}$. 具体步骤为: 在 $250 \mathrm{~mL}$ 的圆底烧瓶中, 加入 $15.3 \mathrm{~g}(0.1$ $\mathrm{mol}$ )对差基硫代苯甲酰胺和 $87 \mathrm{~mL}$ 的无水乙醇, 在冰浴 冷却搅拌下, 滴加 $21.85 \mathrm{~g}(0.1 \mathrm{~mol}) 2$-氯代三氟乙酰乙 
酸乙酯，反应放热，滴加完毕后，升温回流 $24 \mathrm{~h}$, 静置过 夜, 后减压浓缩得橙黄色固液混合物, 加入石油醚, 摚 拌后过滤, 得棕红色固体 $21.6 \mathrm{~g}$, 收率 84\%. m.p. 179 $180{ }^{\circ} \mathrm{C} ;{ }^{1} \mathrm{H}$ NMR (400 MHz, DMSO- $d_{6}$ ) $\delta: 1.32$ (t, $J=7.1$ $\left.\mathrm{Hz}, 3 \mathrm{H}, \mathrm{CH}_{3}\right), 4.25$ (q, J=7.2 Hz, 2H, $\left.\mathrm{CH}_{2}\right), 5.1(\mathrm{~s}, 1 \mathrm{H}$, $\mathrm{OH}), 6.79$ (d, $J=8.4 \mathrm{~Hz}, 2 \mathrm{H}, \mathrm{Ar}-\mathrm{H}), 7.35$ (d, $J=8.3 \mathrm{~Hz}$, $2 \mathrm{H}, \mathrm{Ar}-\mathrm{H})$.

1.2.2 4-(5-乙氧羰基-4-三氟甲基噻唑-2-基)苯氧乙酸 乙酯(4)的制备

参照文献[10]报道的方法合成化合物 4. 具体步骤 为: 向盛有化合物 3a (3.1 mmol)及无水 DMF $(30 \mathrm{~mL})$ 的 圆底烧瓶中加入碳酸钾 $(3.79 \mathrm{mmol})$ 和澳乙酸酯 (3.41 $\mathrm{mmol})$, 于 $80{ }^{\circ} \mathrm{C}$ 反应, TLC 跟踪反应结束后, 减压蒸去 DMF. 向残余的底物中加入 $1 \mathrm{~mol} \cdot \mathrm{L}^{-1} \mathrm{NaOH}(10 \mathrm{~mL})$ 溶 液, 摚拌, 析出大量固体, 抽滤, 干燥得目标产物. 经 ${ }^{1} \mathrm{H}$ NMR 分析确认为目标产物无需提纯直接投料下一 步. ${ }^{1} \mathrm{H}$ NMR (400 MHz, $\left.\mathrm{CDCl}_{3}\right) \delta: 1.30(\mathrm{t}, J=7.0 \mathrm{~Hz}, 3 \mathrm{H}$, $\mathrm{CH}_{3}$ ), 1.37 (t, $J=7.1 \mathrm{~Hz}, 3 \mathrm{H}, \mathrm{CH}_{3}$ ), 4.12 (q, $J=7.0 \mathrm{~Hz}$, $\left.2 \mathrm{H}, \mathrm{CH}_{2}\right), 4.29$ (q, $\left.J=6.8 \mathrm{~Hz}, 2 \mathrm{H}, \mathrm{CH}_{2}\right), 5.18$ (s, $2 \mathrm{H}, \mathrm{CH}_{2}$ ), $5.5(\mathrm{~s}, 1 \mathrm{H},=\mathrm{CH}), 6.97(\mathrm{~d}, J=8.5 \mathrm{~Hz}, 2 \mathrm{H}, \mathrm{Ar}-\mathrm{H}), 7.87(\mathrm{~d}$, $J=8.4 \mathrm{~Hz}, 2 \mathrm{H}, \mathrm{Ar}-\mathrm{H})$.

1.2.3 4-(5-乙氧羰基-4-三氟甲基噻唑-2-基)苯氧乙酸 (5)的制备

在 $25 \mathrm{~mL}$ 圆底烧瓶中, 加入化合物 $4(0.124 \mathrm{~mol})$ 和 乙醇 $(2 \mathrm{~mL})$, 搅拌下加入 $1.25 \mathrm{~mol} \cdot \mathrm{L}^{-1}$ 的氢氧化钠溶液 $(2 \mathrm{~mL})$, 加入完毕后在 $25{ }^{\circ} \mathrm{C}$ 下反应 $2 \mathrm{~h}$, 反应完毕后, 加入水 $(4 \mathrm{~mL})$ 稀释反应液, 然后酸化至 $\mathrm{pH}$ 至 1 , 析出大 量白色固体, 过滤并用水把固体洗两遍, 红外干燥得 0.4 $\mathrm{g}$ 白色固体, 产率 90\%. ${ }^{1} \mathrm{H}$ NMR (400 MHz, DMSO- $d_{6}$ ) $\delta: 1.30$ (t, $\left.J=7.2 \mathrm{~Hz}, 3 \mathrm{H}, \mathrm{CH}_{3}\right), 4.25$ (q, $J=7.2 \mathrm{~Hz}, 2 \mathrm{H}$, $\left.\mathrm{CH}_{2}\right), 5.18\left(\mathrm{~s}, 2 \mathrm{H}, \mathrm{CH}_{2}\right), 6.94(\mathrm{~d}, J=8.6 \mathrm{~Hz}, 2 \mathrm{H}, \mathrm{Ar}-\mathrm{H})$, $7.83(\mathrm{~d}, J=8.6 \mathrm{~Hz}, 2 \mathrm{H}, \mathrm{Ar}-\mathrm{H}), 11.15$ (s, 1H, COOH).

\subsection{4 化合物 $\mathbf{1 a}$ 的制备}

在 $50 \mathrm{~mL}$ 圆底烧瓶中, 将化合物 $\mathbf{5}(0.01 \mathrm{~mol})$ 分批溶 解在 $(\mathrm{COCl})_{2}(0.02 \mathrm{~mol})$ 中, 反应剧烈进行, 加完后, 继 续室温摚拌 $20 \mathrm{~min}$, 然后加热回流 $2 \sim 3 \mathrm{~h}$ 后, 减压浓缩 除去过量的草酰氯和酸气, 后用无水 $\mathrm{CH}_{2} \mathrm{Cl}_{2}(15 \mathrm{~mL})$ 将 其溶解, 在水浴冷却下, 将其滴加到正丁醇 $(0.011 \mathrm{~mol})$ 、 三乙胺 $(0.01 \mathrm{~mol})$ 与无水 $\mathrm{CH}_{2} \mathrm{Cl}_{2}(20 \mathrm{~mL})$ 的混合液中. 滴 加完毕后, 室温继续反应, TLC 跟踪至反应完全后, 减 压浓缩得白色固体, 往其中加入水 $(35 \mathrm{~mL})$, 用乙酸乙酯 萃取后, 无水 $\mathrm{Na}_{2} \mathrm{SO}_{4}$ 干燥, 减压浓缩得黄色固体, 收率 $70 \%$. 结构表征数据见表 4 和 5.
1.2.5 4-(5-羧基-4-取代噻唑-2-基)苯酚(6)的制备

参照文献[11]报道的方法合成化合物 6a 和 $6 \mathbf{b}$. 具体 步骤如下.

在 $250 \mathrm{~mL}$ 的圆底烧瓶中, 加入 2-(4-差基苯基)-4三氟甲基噻唑-5-甲酸乙酯 $(12 \mathrm{~g})$ 和乙醇 $(60 \mathrm{~mL})$, 室温摚 拌下将新配的 $4 \mathrm{~mol} \cdot \mathrm{L}^{-1} \mathrm{NaOH}$ 溶液 $(75 \mathrm{~mL}$ )加入, 升温 回流 $2.5 \mathrm{~h}$, 反应结束. 冷却后缓慢倾入冷水 $(120 \mathrm{~mL})$ 中, 用浓盐酸酸化至 $\mathrm{pH}=1$, 有白色固体析出, 抽滤, 固体 用水洗三遍, 红外灯下烘干, 得 $9.2 \mathrm{~g}$ 白色固体 6a, 收率 $84 \%$. m.p. $188 \sim 189{ }^{\circ} \mathrm{C} ;{ }^{1} \mathrm{H}$ NMR (400 MHz, DMSO- $d_{6}$ ) $\delta: 5.10$ (s, 1H, OH), 6.79 (d, $J=8.4 \mathrm{~Hz}, 2 \mathrm{H}, \mathrm{Ar}-\mathrm{H}), 11.24$ (s, $1 \mathrm{H}, \mathrm{COOH}$ ).

6b 合成方法同化合物 $\mathbf{6 a}$, 定量得白色固体 $\mathbf{6 b}$, m.p. $>200{ }^{\circ} \mathrm{C} ;{ }^{1} \mathrm{HNMR}\left(400 \mathrm{MHz}, \mathrm{DMSO}-d_{6}\right) \delta: 2.65$ (s, $\left.3 \mathrm{H}, \mathrm{CH}_{3}\right), 5.09$ (s, 1H, OH), 6.94 (d, $\left.J=8.6 \mathrm{~Hz}, 2 \mathrm{H}, \mathrm{Ar}-\mathrm{H}\right)$, 7.83 (d, $J=8.6 \mathrm{~Hz}, 2 \mathrm{H}, \mathrm{Ar}-\mathrm{H}), 11.26$ (s, 1H, $\mathrm{COOH}$ ).

1.2.6 2-(4-差基苯基)-4-取代噻唑-5-甲酸衍生物(7) 的制备

在 $50 \mathrm{~mL}$ 圆底烧瓶中, 原料酸 $6(0.01 \mathrm{~mol})$ 与过量 $\mathrm{SOCl}_{2}$ 反应制得酰氯, 然后与 $\mathrm{R}^{2} \mathrm{XH}$ 反应, 制得中间体 7, 相关化合物物理常数及结构表征数据见表 3 .

\subsection{7 化合物 $\mathbf{1 b} \sim \mathbf{1 r}$ 的制备}

参照化合物 $\mathbf{4}$ 的制备方法合成化合物 $\mathbf{1 b} \sim \mathbf{1} \mathbf{r}^{[10]}$, 熔 点、收率和高分辨数据见表 4 , 核磁数据见表 5 .

\subsection{7 化合物 $\mathbf{1 a} \sim 1 \mathbf{r}$ 的生测方法}

油菜平皿法 ${ }^{[12 ~ 15]}$ : 直径 $6 \mathrm{~cm}$ 的培养血中铺好一张 直径 $5.6 \mathrm{~cm}$ 的滤纸, 加入 $2 \mathrm{~mL} 100 \mu \mathrm{g} \cdot \mathrm{mL}^{-1}$ 的供试化合 物溶液, 播种浸种 $4 \sim 6 \mathrm{~h}$ 的油菜种子 15 粒, $28 \pm 1{ }^{\circ} \mathrm{C}$ 下, 黑暗培养 $66 \mathrm{~h}$ 后测定胚根长度. 通过黑暗条件下化合物 对油菜胚根的生长抑制来检测化合物的除草活性. 结果 见表1.

稗草小杯法 ${ }^{[12 \sim 15]}$ : 通过光照条件下对稗草幼苗株 高的生长抑制来检测化合物的除草活性. 测试浓度: 100 $\mu \mathrm{g} \cdot \mathrm{mL}^{-1}$, 活性指标: 株高生长抑制率 (\%). 结果见表 1.

杀菌活性测试: 将一定量药剂溶解在适量 DMF 内, 然后用乳化水稀释至 $50 \mu \mathrm{g} \cdot \mathrm{mL}^{-1}$. 具体过程是: 在无菌 条件下各吸取 $1 \mathrm{~mL}$ 药液注入培养血内, 加入 $9 \mathrm{~mL}$ PDA 培养基, 摇匀后制成 $50 \mu \mathrm{g} \cdot \mathrm{mL}^{-1}$ 含药平板, 以添加 $1 \mathrm{~mL}$ 灭菌水的平板做空白对照. 用直径 $4 \mathrm{~mm}$ 的打孔器沿菌 丝外缘切取菌盘, 移至含药平板上, 每次处理重复三次. 将培养血放在 $(24 \pm 1){ }^{\circ} \mathrm{C}$ 恒温培养箱内培养. $72 \mathrm{~h}$ 后调 查各处理菌盘扩展直径, 求平均值, 与空白对照比较计 算相对抑菌率. 结果见表 2. 
表 3 化合物 7a $\sim 7 \mathbf{f}$ 的物理常数及结构表征数据

Table 3 Melting points, yields, and ${ }^{1} \mathrm{H}$ NMR of compounds $7 \mathbf{a} \sim 7 \mathbf{f}$

\begin{tabular}{|c|c|c|c|c|}
\hline 化合物 & 性状 & m.p. $/{ }^{\circ} \mathrm{C}$ & 收率 $/ \%$ & ${ }^{1} \mathrm{H}$ NMR $\left(400 \mathrm{MHz}, \mathrm{DMSO}-d_{6}\right) \delta$ \\
\hline $7 \mathbf{a}$ & 白色固体 & $196 \sim 197$ & 89 & $\begin{array}{l}3.89\left(\mathrm{~s}, 3 \mathrm{H}, \mathrm{CH}_{3}\right), 5.10(\mathrm{~s}, 1 \mathrm{H}, \mathrm{OH}), 6.94(\mathrm{~d}, J=8.4 \mathrm{~Hz}, 2 \mathrm{H}, \mathrm{Ar}-\mathrm{H}), 7.87(\mathrm{~d}, J=8.4 \mathrm{~Hz}, 2 \mathrm{H} \text {, } \\
\text { Ar-H) }\end{array}$ \\
\hline $7 b$ & 黄色固体 & 133 & 80 & $\begin{array}{l}0.91\left(\mathrm{t}, J=7.1 \mathrm{~Hz}, 3 \mathrm{H}, \mathrm{CH}_{3}\right), 1.33\left(\mathrm{~d}, J=6.4 \mathrm{~Hz}, 3 \mathrm{H}, \mathrm{CH}_{3}\right), 1.45 \sim 1.53\left(\mathrm{~m}, 2 \mathrm{H}, \mathrm{CH}_{2}\right), \\
3.82 \sim 3.86(\mathrm{~m}, 1 \mathrm{H}, \mathrm{CH}), 4.77(\mathrm{~s}, 1 \mathrm{H}, \mathrm{OH}), 6.98(\mathrm{~d}, J=8.1 \mathrm{~Hz}, 2 \mathrm{H}, \mathrm{Ar}-\mathrm{H}), 7.85(\mathrm{~d}, J=8.1 \\
\mathrm{Hz}, 2 \mathrm{H}, \mathrm{Ar}-\mathrm{H})\end{array}$ \\
\hline $7 \mathrm{c}$ & 黄色固体 & $167 \sim 168$ & 75 & $\begin{array}{l}1.15\left(\mathrm{~d}, J=6.6 \mathrm{~Hz}, 6 \mathrm{H}, \mathrm{CH}_{3}\right), 3.99 \sim 4.03(\mathrm{~m}, 1 \mathrm{H}, \mathrm{CH}), 5.10(\mathrm{~s}, 1 \mathrm{H}, \mathrm{OH}), 6.94(\mathrm{~d}, J=8.5 \\
\mathrm{Hz}, 2 \mathrm{H}, \mathrm{Ar}-\mathrm{H}), 7.84(\mathrm{~d}, J=8.5 \mathrm{~Hz}, 2 \mathrm{H}, \mathrm{Ar}-\mathrm{H})\end{array}$ \\
\hline $7 d$ & 黄色固体 & $198 \sim 199$ & 94 & $\begin{array}{l}2.70\left(\mathrm{~s}, 3 \mathrm{H}, \mathrm{CH}_{3}\right), 4.62\left(\mathrm{~s}, 3 \mathrm{H}, \mathrm{CH}_{3}\right), 5.10(\mathrm{~s}, 1 \mathrm{H}, \mathrm{OH}), 6.88(\mathrm{~d}, J=8.8 \mathrm{~Hz}, 2 \mathrm{H}, \mathrm{Ar}-\mathrm{H}), 7.84 \\
(\mathrm{~d}, J=8.8 \mathrm{~Hz}, 2 \mathrm{H}, \mathrm{Ar}-\mathrm{H})\end{array}$ \\
\hline $7 e$ & 黄色固体 & $177 \sim 178$ & 91 & $\begin{array}{l}0.91\left(\mathrm{t}, J=7.1 \mathrm{~Hz}, 3 \mathrm{H}, \mathrm{CH}_{3}\right), 133\left(\mathrm{~d}, J=6.4 \mathrm{~Hz}, 3 \mathrm{H}, \mathrm{CH}_{3}\right), 1.50 \sim 1.54\left(\mathrm{~m}, 2 \mathrm{H}, \mathrm{CH}_{2}\right), 2.51 \\
\left(\mathrm{~s}, 3 \mathrm{H}, \mathrm{CH}_{3}\right), 3.72 \sim 3.78(\mathrm{~m}, 1 \mathrm{H}, \mathrm{CH}), 4.80(\mathrm{~s}, 1 \mathrm{H}, \mathrm{OH}), 6.95(\mathrm{~d}, J=8.2 \mathrm{~Hz}, 2 \mathrm{H}, \mathrm{Ar}-\mathrm{H}), \\
7.86(\mathrm{~d}, J=8.1 \mathrm{~Hz}, 2 \mathrm{H}, \mathrm{Ar}-\mathrm{H})\end{array}$ \\
\hline $7 f$ & 黄色固体 & $198 \sim 199$ & 83 & $\begin{array}{l}1.05\left(\mathrm{~d}, J=6.6 \mathrm{~Hz}, 6 \mathrm{H}, \mathrm{CH}_{3}\right), 2.51\left(\mathrm{~s}, 3 \mathrm{H}, \mathrm{CH}_{3}\right), 3.92 \sim 4.06(\mathrm{~m}, 1 \mathrm{H}, \mathrm{CH}), 5.10(\mathrm{~s}, 1 \mathrm{H}, \mathrm{OH}), \\
6.91(\mathrm{~d}, J=8.4 \mathrm{~Hz}, 2 \mathrm{H}, \mathrm{Ar}-\mathrm{H}), 7.82(\mathrm{~d}, J=8.4 \mathrm{~Hz}, 2 \mathrm{H}, \mathrm{Ar}-\mathrm{H})\end{array}$ \\
\hline
\end{tabular}

表 4 化合物 $1 \mathrm{a} \sim 1 \mathrm{r}$ 的物理常数及高分辨质谱数据

Table 4 Melting points, yields, and HRMS of compounds $\mathbf{1 a} \sim \mathbf{1 r}$

\begin{tabular}{ccccc}
\hline 化合物 & 性状 & m.p. $/{ }^{\circ} \mathrm{C}$ & 产率/\% & HRMS $[\mathrm{M}+\mathrm{Na}]^{+}(\mathrm{calcd})$ \\
\hline $\mathbf{1 a}$ & 白色固体 & $131 \sim 132$ & 70.0 & $344.0569(344.0563)$ \\
$\mathbf{1 b}$ & 白色固体 & $123 \sim 124$ & 83.6 & $358.0717(358.0720)$ \\
$\mathbf{1 c}$ & 白色固体 & $74 \sim 75$ & 75.2 & $386.1036(386.1033)$ \\
$\mathbf{1 d}$ & 白色固体 & $59 \sim 60$ & 82.5 & $420.1834(420.1839)$ \\
$\mathbf{1 e}$ & 白色固体 & $106 \sim 107$ & 55.7 & $358.0726(358.0720)$ \\
$\mathbf{1 f}$ & 白色固体 & $87 \sim 88$ & 41.5 & $372.0875(372.0876)$ \\
$\mathbf{1 g}$ & 白色固体 & $71 \sim 72$ & 47.8 & $400.1196(400.1189)$ \\
$\mathbf{1 h}$ & 白色固体 & $53 \sim 54$ & 72.0 & $434.1995(434.1996)$ \\
$\mathbf{1 i}$ & 白色固体 & $148 \sim 149$ & 58.1 & $371.1032(371.1036)$ \\
$\mathbf{1 j}$ & 白色固体 & $124 \sim 125$ & 65.8 & $389.1538(389.1541)$ \\
$\mathbf{1 k}$ & 白色固体 & $113 \sim 114$ & 93.9 & $483.2285(483.2288)$ \\
$\mathbf{1 I}$ & 白色固体 & $155 \sim 156$ & 84.9 & $425.0750(425.0753)$ \\
$\mathbf{1 m}$ & 白色固体 & $129 \sim 130$ & 15.2 & $415.0947(415.0945)$ \\
$\mathbf{1 n}$ & 白色固体 & $72 \sim 73$ & 38.0 & $523.1845(523.1849)$ \\
$\mathbf{1 0}$ & 白色固体 & $158 \sim 159$ & 67.3 & $415.0943(415.0945)$ \\
$\mathbf{1 p}$ & 白色固体 & $137 \sim 138$ & 26.5 & $398.0284(398.0280)$ \\
$\mathbf{1 q}$ & 白色固体 & $72 \sim 73$ & 65.0 & $440.0742(440.0750)$ \\
$\mathbf{1 r}$ & 白色固体 & $62 \sim 63$ & 32.0 & $454.0908(454.0907)$ \\
\hline
\end{tabular}

表 5 化合物 $1 \mathrm{a} \sim 1 \mathrm{r}$ 的核磁数据

Table $5{ }^{1} \mathrm{H}$ NMR of compounds $\mathbf{1 a} \sim \mathbf{1 r}$

\begin{tabular}{|c|c|}
\hline Compd. & ${ }^{1} \mathrm{H} \mathrm{NMR}\left(400 \mathrm{MHz}, \mathrm{CDCl}_{3}\right) \delta$ \\
\hline $1 \mathbf{a}$ & $\begin{array}{l}2.76\left(\mathrm{~s}, 3 \mathrm{H}, \mathrm{CH}_{3}\right), 3.82\left(\mathrm{~s}, 3 \mathrm{H}, \mathrm{CH}_{3}\right), 3.88\left(\mathrm{~s}, 3 \mathrm{H}, \mathrm{CH}_{3}\right), 4.70\left(\mathrm{~s}, 2 \mathrm{H}, \mathrm{CH}_{2}\right), 6.98(\mathrm{~d}, J=7.9 \mathrm{~Hz}, 2 \mathrm{H}, \mathrm{Ar}-\mathrm{H}), 7.91(\mathrm{~d}, J=7.9 \mathrm{~Hz}, \\
2 \mathrm{H}, \mathrm{Ar}-\mathrm{H})\end{array}$ \\
\hline $1 \mathbf{b}$ & $\begin{array}{l}1.24\left(\mathrm{t}, J=5.9 \mathrm{~Hz}, 3 \mathrm{H}, \mathrm{CH}_{3}\right), 2.69\left(\mathrm{~s}, 3 \mathrm{H}, \mathrm{CH}_{3}\right), 3.81\left(\mathrm{~s}, 3 \mathrm{H}, \mathrm{CH}_{3}\right), 4.22\left(\mathrm{q}, J=6.5 \mathrm{~Hz}, 2 \mathrm{H}, \mathrm{CH}_{2}\right), 4.60\left(\mathrm{~s}, 2 \mathrm{H}, \mathrm{CH}_{2}\right), 6.89(\mathrm{~d}, \\
J=8.3 \mathrm{~Hz}, 2 \mathrm{H}, \mathrm{Ar}-\mathrm{H}), 7.84(\mathrm{~d}, J=8.3 \mathrm{~Hz}, 2 \mathrm{H}, \mathrm{Ar}-\mathrm{H})\end{array}$ \\
\hline $1 \mathrm{c}$ & $\begin{array}{l}0.94\left(\mathrm{t}, J=7.3 \mathrm{~Hz}, 3 \mathrm{H}, \mathrm{CH}_{3}\right), 1.33 \sim 1.4\left(\mathrm{~m}, 2 \mathrm{H}, \mathrm{CH}_{2}\right), 1.62 \sim 1.70\left(\mathrm{~m}, 2 \mathrm{H}, \mathrm{CH}_{2}\right), 2.79\left(\mathrm{~s}, 3 \mathrm{H}, \mathrm{CH}_{3}\right), 3.91\left(\mathrm{~s}, 3 \mathrm{H}, \mathrm{CH}_{3}\right), 4.24 \\
\left(\mathrm{t}, J=6.6 \mathrm{~Hz}, 2 \mathrm{H}, \mathrm{CH}_{2}\right), 4.71\left(\mathrm{~s}, 2 \mathrm{H}, \mathrm{CH}_{2}\right), 6.98(\mathrm{~d}, J=8.2 \mathrm{~Hz}, 2 \mathrm{H}, \mathrm{Ar}-\mathrm{H}), 7.93(\mathrm{~d}, J=8.2 \mathrm{~Hz}, 2 \mathrm{H}, \mathrm{Ar}-\mathrm{H})\end{array}$ \\
\hline 1d & $\begin{array}{l}0.88\left(\mathrm{t}, J=6.9 \mathrm{~Hz}, 3 \mathrm{H}, \mathrm{CH}_{3}\right), 1.21 \sim 1.29\left(\mathrm{~m}, 10 \mathrm{H}, \mathrm{CH}_{2}\right), 1.62 \sim 1.65\left(\mathrm{~m}, 2 \mathrm{H}, \mathrm{CH}_{2}\right), 2.76\left(\mathrm{~s}, 3 \mathrm{H}, \mathrm{CH}_{3}\right), 3.88\left(\mathrm{~s}, 3 \mathrm{H}, \mathrm{CH}_{3}\right), 4.22 \\
\left(\mathrm{t}, J=6.6 \mathrm{~Hz}, 2 \mathrm{H}, \mathrm{CH}_{2}\right), 4.69\left(\mathrm{~s}, 2 \mathrm{H}, \mathrm{CH}_{2}\right), 6.96(\mathrm{~d}, J=8.5 \mathrm{~Hz}, 2 \mathrm{H}, \mathrm{Ar}-\mathrm{H}), 7.91(\mathrm{~d}, J=8.5 \mathrm{~Hz}, 2 \mathrm{H}, \mathrm{Ar}-\mathrm{H})\end{array}$ \\
\hline
\end{tabular}




Compd. $\quad{ }^{1} \mathrm{H}$ NMR $\left(400 \mathrm{MHz}, \mathrm{CDCl}_{3}\right) \delta$

$1.38\left(\mathrm{t}, J=7.1 \mathrm{~Hz}, 3 \mathrm{H}, \mathrm{CH}_{3}\right), 2.76\left(\mathrm{~s}, 3 \mathrm{H}, \mathrm{CH}_{3}\right), 3.82\left(\mathrm{~s}, 2 \mathrm{H}, \mathrm{CH}_{2}\right), 4.35\left(\mathrm{q}, J=7.1 \mathrm{~Hz}, 2 \mathrm{H}, \mathrm{CH}_{2}\right), 4.69\left(\mathrm{~s}, 2 \mathrm{H}, \mathrm{CH}_{2}\right), 6.95(\mathrm{~d}$, $J=8.6 \mathrm{~Hz}, 2 \mathrm{H}, \mathrm{Ar}-\mathrm{H}), 7.91(\mathrm{~d}, J=8.6 \mathrm{~Hz}, 2 \mathrm{H}, \mathrm{Ar}-\mathrm{H})$

1f

$1.32\left(\mathrm{t}, J=7.1 \mathrm{~Hz}, 3 \mathrm{H}, \mathrm{CH}_{3}\right), 1.40\left(\mathrm{t}, J=7.1 \mathrm{~Hz}, 3 \mathrm{H}, \mathrm{CH}_{3}\right), 2.76\left(\mathrm{~s}, 3 \mathrm{H}, \mathrm{CH}_{3}\right), 4.29\left(\mathrm{q}, J=7.2 \mathrm{~Hz}, 2 \mathrm{H}, \mathrm{CH}_{2}\right), 4.35(\mathrm{q}, J=7.0$ $\mathrm{Hz}, 2 \mathrm{H}), 4.68\left(\mathrm{~s}, 2 \mathrm{H}, \mathrm{CH}_{2}\right), 6.96(\mathrm{~d}, J=8.5 \mathrm{~Hz}, 2 \mathrm{H}, \mathrm{Ar}-\mathrm{H}), 7.91(\mathrm{~d}, J=8.5 \mathrm{~Hz}, 2 \mathrm{H}, \mathrm{Ar}-\mathrm{H})$

$0.93\left(\mathrm{t}, J=7.3 \mathrm{~Hz}, 3 \mathrm{H}, \mathrm{CH}_{3}\right), 1.35 \sim 1.40\left(\mathrm{~m}, 2 \mathrm{H}, \mathrm{CH}_{2}\right), 1.39\left(\mathrm{t}, J=7.1 \mathrm{~Hz}, 3 \mathrm{H}, \mathrm{CH}_{3}\right), 1.61 \sim 1.68\left(\mathrm{~m}, 2 \mathrm{H}, \mathrm{CH}_{2}\right), 2.76(\mathrm{~s}, 3 \mathrm{H}$,

$\left.1 \mathrm{C} \mathrm{CH}_{3}\right), 4.23\left(\mathrm{t}, J=6.7 \mathrm{~Hz}, 2 \mathrm{H}, \mathrm{CH}_{2}\right), 4.36\left(\mathrm{q}, J=7.1 \mathrm{~Hz}, 2 \mathrm{H}, \mathrm{CH}_{2}\right), 4.69\left(\mathrm{~s}, 2 \mathrm{H}, \mathrm{CH}_{2}\right), 6.96(\mathrm{~d}, J=8.5 \mathrm{~Hz}, 2 \mathrm{H}, \mathrm{Ar}-\mathrm{H}), 7.91(\mathrm{~d}$, $J=8.5 \mathrm{~Hz}, 2 \mathrm{H}, \mathrm{Ar}-\mathrm{H})$

$0.85\left(\mathrm{t}, J=7.1 \mathrm{~Hz}, 3 \mathrm{H}, \mathrm{CH}_{3}\right), 1.10 \sim 1.23\left(\mathrm{~m}, 10 \mathrm{H}, \mathrm{CH}_{2}\right), 1.31\left(\mathrm{t}, J=7.1 \mathrm{~Hz}, 3 \mathrm{H}, \mathrm{CH}_{3}\right), 1.53 \sim 1.59\left(\mathrm{~m}, 2 \mathrm{H}, \mathrm{CH}_{2}\right), 4.13(\mathrm{t}, J=$

1h $\left.\quad 6.7 \mathrm{~Hz}, 2 \mathrm{H}, \mathrm{CH}_{2}\right), 4.26\left(\mathrm{q}, J=7.1 \mathrm{~Hz}, 2 \mathrm{H}, \mathrm{CH}_{2}\right), 4.61\left(\mathrm{~s}, 2 \mathrm{H}, \mathrm{CH}_{2}\right), 6.84(\mathrm{~d}, J=8.8 \mathrm{~Hz}, 2 \mathrm{H}, \mathrm{Ar}-\mathrm{H}), 7.84(\mathrm{~d}, J=8.8 \mathrm{~Hz}, 2 \mathrm{H}$, Ar-H)

$1.25\left(\mathrm{t}, J=6.6 \mathrm{~Hz}, 6 \mathrm{H}, \mathrm{CH}_{3}\right), 4.21 \sim 4.70(\mathrm{~m}, 1 \mathrm{H}, \mathrm{CH}), 5.59(\mathrm{~d}, J=7.4 \mathrm{~Hz}, 1 \mathrm{H}, \mathrm{NH}), 6.97(\mathrm{~d}, J=8.5 \mathrm{~Hz}, 2 \mathrm{H}, \mathrm{Ar}-\mathrm{H}), 7.88(\mathrm{~d}$, $J=8.5 \mathrm{~Hz}, 2 \mathrm{H}, \mathrm{Ar}-\mathrm{H})$

$0.86\left(\mathrm{t}, J=7.2 \mathrm{~Hz}, 3 \mathrm{H}, \mathrm{CH}_{3}\right), 1.19\left(\mathrm{~d}, J=6.3 \mathrm{~Hz}, 6 \mathrm{H}, \mathrm{CH}_{3}\right), 1.26 \sim 1.31\left(\mathrm{~m}, 2 \mathrm{H}, \mathrm{CH}_{2}\right), 1.53 \sim 1.59\left(\mathrm{~m}, 2 \mathrm{H}, \mathrm{CH}_{2}\right), 2.64(\mathrm{~s}, 3 \mathrm{H}$,

1j $\left.\quad \mathrm{CH}_{3}\right), 4.16\left(\mathrm{t}, J=6.3 \mathrm{~Hz}, 2 \mathrm{H}, \mathrm{CH}_{2}\right), 4.16 \sim 4.19(\mathrm{~m}, 1 \mathrm{H}, \mathrm{CH}), 4.60\left(\mathrm{~s}, 2 \mathrm{H}, \mathrm{CH}_{2}\right), 5.52(\mathrm{~d}, J=6.5 \mathrm{~Hz}, 1 \mathrm{H}, \mathrm{NH}), 6.88(\mathrm{~d}, J=8.4$ $\mathrm{Hz}, 2 \mathrm{H}, \mathrm{Ar}-\mathrm{H}), 7.79$ (d, $J=8.4 \mathrm{~Hz}, 2 \mathrm{H}, \mathrm{Ar}-\mathrm{H})$

$0.88\left(\mathrm{t}, J=6.1 \mathrm{~Hz}, 3 \mathrm{H}, \mathrm{CH}_{3}\right), 0.99\left(\mathrm{t}, J=7.0 \mathrm{~Hz}, 3 \mathrm{H}, \mathrm{CH}_{3}\right), 1.25 \sim 1.27\left(\mathrm{~m}, 8 \mathrm{H}, \mathrm{CH}_{2}\right), 1.26\left(\mathrm{~d}, J=8.0 \mathrm{~Hz}, 3 \mathrm{H}, \mathrm{CH}_{3}\right), 1.58 \sim$

$1 \mathbf{k} \quad 1.65\left(\mathrm{~m}, 6 \mathrm{H}, \mathrm{CH}_{2}\right), 2.73\left(\mathrm{~s}, 3 \mathrm{H}, \mathrm{CH}_{3}\right), 4.22\left(\mathrm{t}, J=6.7 \mathrm{~Hz}, 2 \mathrm{H}, \mathrm{CH}_{2}\right), 4.06 \sim 4.13(\mathrm{~m}, 1 \mathrm{H}, \mathrm{CH}), 4.69\left(\mathrm{~s}, 2 \mathrm{H}, \mathrm{CH}_{2}\right), 5.52(\mathrm{~d}, J=$ $5.3 \mathrm{~Hz}, 1 \mathrm{H}, \mathrm{NH}), 6.96(\mathrm{~d}, J=8.5 \mathrm{~Hz}, 2 \mathrm{H}, \mathrm{Ar}-\mathrm{H}), 7.45(\mathrm{~d}, J=8.5 \mathrm{~Hz}, 2 \mathrm{H}, \mathrm{Ar}-\mathrm{H})$

$1.2\left(\mathrm{~d}, J=6.6 \mathrm{~Hz}, 6 \mathrm{H}, \mathrm{CH}_{3}\right), 3.76\left(\mathrm{~s}, 3 \mathrm{H}, \mathrm{CH}_{3}\right), 4.14 \sim 4.22(\mathrm{~m}, 1 \mathrm{H}, \mathrm{CH}), 4.64\left(\mathrm{~s}, 2 \mathrm{H}, \mathrm{CH}_{2}\right), 5.90(\mathrm{~d}, J=7.5 \mathrm{~Hz}, 1 \mathrm{H}, \mathrm{NH}), 6.90$ (d, $J=8.8 \mathrm{~Hz}, 2 \mathrm{H}, \mathrm{Ar}-\mathrm{H}), 7.82(\mathrm{~d}, J=8.8 \mathrm{~Hz}, 2 \mathrm{H}, \mathrm{Ar}-\mathrm{H})$

$1.20\left(\mathrm{~d}, J=6.6 \mathrm{~Hz}, 6 \mathrm{H}, \mathrm{CH}_{3}\right), 4.23\left(\mathrm{q}, J=7.1 \mathrm{~Hz}, 2 \mathrm{H}, \mathrm{CH}_{2}\right), 4.14 \sim 4.19(\mathrm{~m}, 1 \mathrm{H}, \mathrm{CH}), 4.61\left(\mathrm{~s}, 2 \mathrm{H}, \mathrm{CH}_{2}\right), 5.92(\mathrm{~d}, J=6.3 \mathrm{~Hz}$, $1 \mathrm{H}, \mathrm{NH}), 6.90(\mathrm{~d}, J=8.7 \mathrm{~Hz}, 2 \mathrm{H}, \mathrm{Ar}-\mathrm{H}), 7.81(\mathrm{~d}, J=8.7 \mathrm{~Hz}, 2 \mathrm{H}, \mathrm{Ar}-\mathrm{H})$

$0.87\left(\mathrm{t}, J=6.9 \mathrm{~Hz}, 3 \mathrm{H}, \mathrm{CH}_{3}\right), 1.21 \sim 1.29\left(\mathrm{~m}, 10 \mathrm{H}, \mathrm{CH}_{2}\right), 1.25\left(\mathrm{~d}, J=6.9 \mathrm{~Hz}, 3 \mathrm{H}, \mathrm{CH}_{3}\right), 1.63 \sim 1.69\left(\mathrm{~m}, 2 \mathrm{H}, \mathrm{CH}_{2}\right), 4.21(\mathrm{t}, J=$

1n $\left.7.1 \mathrm{~Hz}, 2 \mathrm{H}, \mathrm{CH}_{2}\right), 4.22 \sim 4.28(\mathrm{~m}, \mathrm{H}, \mathrm{CH}), 4.68\left(\mathrm{~s}, 2 \mathrm{H}, \mathrm{CH}_{2}\right), 6.07(\mathrm{~d}, J=6.3 \mathrm{~Hz}, 1 \mathrm{H}, \mathrm{NH}), 6.96(\mathrm{~d}, J=8.6 \mathrm{~Hz}, 2 \mathrm{H}, \mathrm{Ar}-\mathrm{H}), 7.87$ (d, $J=8.6 \mathrm{~Hz}, 2 \mathrm{H}, \mathrm{Ar}-\mathrm{H})$

$10 \quad 0.99$ (t, $\left.J=7.4 \mathrm{~Hz}, 3 \mathrm{H}, \mathrm{CH}_{3}\right), 1.24\left(\mathrm{~d}, J=6.6 \mathrm{~Hz}, 3 \mathrm{H}, \mathrm{CH}_{3}\right), 1.54 \sim 1.64\left(\mathrm{~m}, 2 \mathrm{H}, \mathrm{CH}_{2}\right), 3.83\left(\mathrm{~s}, 3 \mathrm{H}, \mathrm{CH}_{3}\right), 3.93 \sim 4.14(\mathrm{~m}, 1 \mathrm{H}$, $\mathrm{CH}), 4.70\left(\mathrm{~s}, 2 \mathrm{H}, \mathrm{CH}_{2}\right), 5.60$ (d, J=7.2 Hz, 1H, NH), 6.98 (d, J=8.9 Hz, 2H, Ar-H), 7.90 (d, J=8.9 Hz, 2H, Ar-H)

$1 p \quad 3.67\left(\mathrm{~s}, 3 \mathrm{H}, \mathrm{CH}_{3}\right), 3.88\left(\mathrm{~s}, 3 \mathrm{H}, \mathrm{CH}_{3}\right), 4.63\left(\mathrm{~s}, 2 \mathrm{H}, \mathrm{CH}_{2}\right), 6.82(\mathrm{~d}, J=8.7 \mathrm{~Hz}, 2 \mathrm{H}, \mathrm{Ar}-\mathrm{H}), 7.85(\mathrm{~d}, J=8.7 \mathrm{~Hz}, 2 \mathrm{H}, \mathrm{Ar}-\mathrm{H})$ $0.94\left(\mathrm{t}, J=7.4 \mathrm{~Hz}, 3 \mathrm{H}, \mathrm{CH}_{3}\right), 1.31 \sim 1.37\left(\mathrm{~m}, 2 \mathrm{H}, \mathrm{CH}_{2}\right), 1.64 \sim 1.68\left(\mathrm{~m}, 2 \mathrm{H}, \mathrm{CH}_{2}\right), 3.95\left(\mathrm{~s}, 3 \mathrm{H}, \mathrm{CH}_{3}\right), 4.24(\mathrm{t}, J=6.7 \mathrm{~Hz}, 2 \mathrm{H}$,

$\left.1 \mathrm{q} \quad \mathrm{CH}_{2}\right), 4.70\left(\mathrm{~s}, 2 \mathrm{H}, \mathrm{CH}_{2}\right), 6.98(\mathrm{~d}, J=8.7 \mathrm{~Hz}, 2 \mathrm{H}, \mathrm{Ar}-\mathrm{H}), 7.94(\mathrm{~d}, J=8.7 \mathrm{~Hz}, 2 \mathrm{H}, \mathrm{Ar}-\mathrm{H})$

$0.94\left(\mathrm{t}, J=7.4 \mathrm{~Hz}, 3 \mathrm{H}, \mathrm{CH}_{3}\right), 1.31 \sim 1.37\left(\mathrm{~m}, 2 \mathrm{H}, \mathrm{CH}_{2}\right), 1.41\left(\mathrm{t}, J=7.13 \mathrm{~Hz}, 3 \mathrm{H}, \mathrm{CH}_{3}\right), 1.65 \sim 1.68\left(\mathrm{~m}, 2 \mathrm{H}, \mathrm{CH}_{2}\right), 4.24(\mathrm{t}, J=$ $\left.1 \mathbf{r} 6.6 \mathrm{~Hz}, 2 \mathrm{H}, \mathrm{CH}_{2}\right), 4.42\left(\mathrm{q}, J=7.1 \mathrm{~Hz}, 2 \mathrm{H}, \mathrm{CH}_{2}\right), 4.70\left(\mathrm{~s}, 2 \mathrm{H}, \mathrm{CH}_{2}\right), 6.98(\mathrm{~d}, J=8.7 \mathrm{~Hz}, 2 \mathrm{H}, \mathrm{Ar}-\mathrm{H}), 7.94(\mathrm{~d}, J=8.7 \mathrm{~Hz}, 2 \mathrm{H}$, Ar-H)

\section{References}

[1] Zhong, B.; Liu, C.-L.; Li, Z.-M. New Pestic. 2004, 2, 7 (in Chinese).

(钟滨, 刘长令, 李正名, 新农药, 2004, 2, 7.)

[2] Yan, F.-Y.; Liu, D.-Q.; Guan, A.-Y.; Liu, C.-L. Agrochemicals 2003, 42, 46 (in Chinese).

(颜范勇, 刘冬青, 关爱荣, 刘长令, 农药, 2003, 42, 46.)

[3] Liu, C.-L. The Collection of New Pesticide Research, Chemical Industry Press, Beijing, 2002, p. 132 (in Chinese).

(刘长令, 新农药研究开发文集, 化学工业出版社, 北京, 2002, p. 132.)

[4] Bartroli, J.; Turmo, E.; Alguero, M.; Boncompte, E.; Vericat, M. L.; Conte, L.; Ramis, J.; Merlos, M.; Garcia-Rafanell, J.; Forn, J. J. Med. Chem. 1998, 41, 1855.

[5] Shuji, M.; Hiroyuki, K.; Takami, Y.; Menetsugu, M.; Junji, K.; Noriaki, K.; Mitsuru, I. JP 10067759, 1998 [Chem. Abstr. 1998, 128,
217365].

[6] Tang, C.-C.; Li, Y.-C.; Chen, B.; Yang, H.-Z.; Jin, G.-Y. Pesticide Chemistry, Nankai University Press, Tianjin, 1998, pp. 488 454 (in Chinese).

(唐除痴，李显昶，陈涁，杨华铮，金桂玉，农药化学，南开大学 出版社, 天津, 1998, pp. 488 454.)

[7] Liu, C.-L. World Pesticides, Chemical Industry Press, Beijing, 2002, pp. 200 202 (in Chinese).

(刘长令, 世界农药, 化学工业出版社, 北京, 2002, pp. 200 202.)

[8] Dmitri, V. S.; Mikhail, V.; Vyacheslav, Y. S.; Helma, W.; Olesya, K.; Vera, V.; Nikolay, E. S.; Valentine, G. N.; Enno, L.; GerdVolker, R. Tetrahedron 2009, 65, 7538.

[9] Robbins, T. A.; Zhu, H.; Shao, J. US 20050027128, 2005 [Chem. Abstr. 2005, 142, 176830].

[10] Varma, R. S.; Chauhan, S.; Prasad, C. R. Indian J. Chem., Sect. B 1988, 27B, 438. 
[11] Michael, L. S.; Véronique, B.; Anne-Bénédict, B.; Thierry, B.; Andrew, G. B.; Frédéric, D.; Marie-Claire, F.; Marie-Hélène, F.; Françoise, J. G.; Didier, A. G.; Millard, H. L.; Alain, L.; Christelle Le G.; Jean, M. L.; Valerie, G. M.; Van-Loc, N.; Edwige, N.; Vipul, P.; Annie, P.; Olivier, P.; Danig, P.; Florent, P.; Géraldine, P.; Cécile, B. R.; Michael, S.; Jérôme, T.; Xu, E. H.; Xu, R. X.; Pascal, M. P. J. Med. Chem. 2007, 50, 685.

[12] Zhu, Y.-Q.; Liu, C.; Zhu, R.; Zhang, J.; Yuan, Y.-W.; Zou, X.-M.; Hu, F.-Z.; Yang, H.-Z. Chin. J. Org. Chem. 2010, 30, 1088 (in Chinese).

(朱有全, 刘翠, 朱然, 张金, 袁燕伟, 邹小毛, 胡方中, 杨华铮, 有机化学, 2010, 30, 1088.)
[13] Zhu, Y.-Q.; Zhu, R.; Yuan, Y.-W.; Zhang, J.; Zou, X.-M.; Hu, F.-Z.; Yang, H.-Z. Chin. J. Org. Chem. 2010, 30, 1207 (in Chinese). (朱有全, 朱然, 袁燕伟, 张金, 邹小毛, 胡方中, 杨华铮, 有机 化学, 2010, 30, 1207.)

[14] Zhu, Y.-Q.; Liu, W.-M.; Liu, B.; Hu, F.-Z.; Zhu, R.; Zou, X.-M.; Yang, H.-Z. Chin. J. Org. Chem. 2009, 29, 638 (in Chinese). (朱有全, 刘卫敏, 刘斌, 胡方中, 朱然, 邹小毛, 杨华铮, 有机 化学, 2009, 29, 638.)

[15] Zhu, Y.-Q.; Cheng, J.; Zou, X.-M.; Hu, F.-Z.; Xiao, Y.-H.; Yang, H.-Z. Chin. J. Org. Chem. 2008, 28, 1044 (in Chinese). (朱有全, 程佳, 邹小毛, 胡方中, 肖玉虹, 杨华铮, 有机化学, 2008, 28, 1044.)

(Cheng, F.) 